\title{
Anticonvulsants for the Treatment of Hemichorea after Surgical Revascularization in Adolescent Patients with Moyamoya Disease
}

\author{
Ga Hee Lee, MD', Dong Hyun Kim, MD', Hyeon Seon Park, MD², Woo Ri Jang, MD³, Young Se Kwon, MD \\ 'Department of Pediatrics, Inha University Hospital, Incheon, Korea \\ ${ }^{2}$ Department of Neurosurgery, Inha University Hospital, Incheon, Korea \\ ${ }^{3}$ Inha University Hospital Rare Genetic Disease Center, Incheon, Korea
}

Received: September 29, 2020

Revised: December 2, 2020

Accepted: December 2, 2020

Corresponding author:

Young Se Kwon, MD

Department of Pediatrics, Inha University School of Medicine, 27

Inhang-ro, Jung-gu, Incheon

22332, Korea

Tel: +82-32-890-2843

Fax: +82-32-890-2844

E-mail:ysped@inha.ac.kr
Chorea is uncommon clinical presentation in moyamoya disease (MMD); it can be present in $3 \%$ to $6 \%$ of patients [1]. Although the mechanism of development of chorea is unclear, ischemia of the basal ganglia, which could lead to post stroke abnormal movements is considered a dominant mechanism. A majority of chorea symptoms improve within 1 year of surgical revascularization of the ischemic region. However, it is very rare that chorea develops after surgical revascularization. We report two adolescent cases of new-onset hemichorea after diagnosis of $\mathrm{MMD}$ and bilateral bypass surgery.

An 11-year-old boy (Patient A) had undergone bilateral bypass surgery for MMD. Four years after the bypass surgery, the patient was referred to our department for new-onset hemichorea. Intermittent tremors began in the right arm and gradually worsened to become sporadic irregular, dance-like movements of hemichorea, which resulted in twisting movements. One week later, the hemichorea progressed to involve the right leg, which made walking difficult.

A 16-year-old girl (Patient B) had undergone bilateral bypass surgery for MMD. Two years after the bypass surgery, she developed new trem- ors in the right hand. The tremors worsend to become invaluntary movement of right arm.

We performed bilateral internal carotid artery angiography and perfusion magnetic resonance imaging (MRI) of the brain with gadolinium. At the first diagnosis, MRI of both patients revealed prominent network of collateral vessels in the basal ganglia as well as stenosis of the internal carotid artery. However, it was confirmed that there were no new lesions in the blood vessels and there was no reduction in brain diffusion and blood flow reserve compared with the previous findings (Figs. 1 and 2). We approached the cause of chorea not from the perspective of new brain vasculopathy but from that of the neurotransmission system. We considered the age variation of neuronal structure in the basal ganglia was the cause of chorea, which was not present at the time of diagnosis in children with MMD, occurs newly in adolescence.

Neurons in the striatum in the basal nucleus exclusively regulate the activation and suppression of spontaneous behavior through direct and indirect pathways, respectively [2]. These pathways are regulated by the neurotransmitter dopamine. The possible pathophysiological mecha-

Copyright (C) 2021 Korean Child Neurology Society

This is an Open Access article distributed under the terms of the Creative Commons Attribution Non-Commercial License (http://creativecommons.org/licenses/by-nc/4.0/) which permits unrestricted non-commercial use, distribution, and reproduction in any medium, provided the original work is properly cited. 

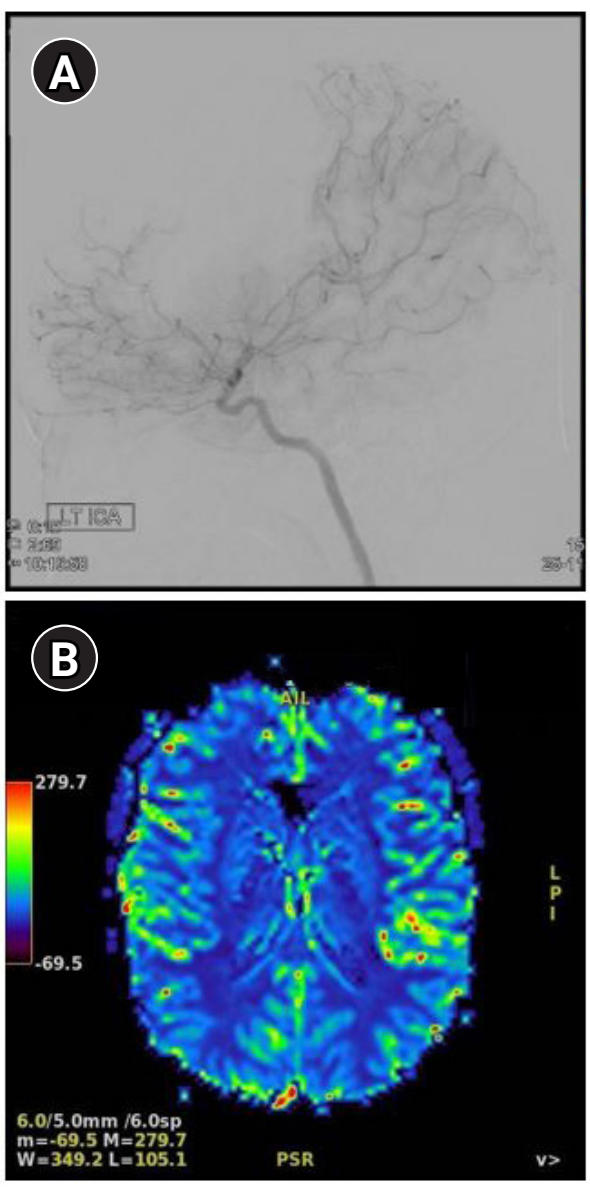

Fig. 1. Images obtained in patient A after surgical revascularization. (A) Intertal carotid artery (ICA) angiography exposed the complete occlusion of right ICA, left anterior cerebral artery with basal collaterals. Good neovascularization was confirmed through indirect bypass surgery. (B) Brain perfusion magnetic resonance imaging showed no perfusion abnormality in brain parenchyma.

nism of chorea appears to be inactivity of the indirect pathways in the basal ganglia-thalamocortical motor circuits [3]. In the presence of striatal lesions, the inhibition to the lateral globus pallidus is weakened and the inhibition to the subthalamic nucleus is activated. Suppressed subthalamic nucleus results in less inhibition of the thalamus by the globus pallidus pars interna, thus, resulting in unwanted movements. This is the currently accepted hypothesis for basal ganglia dysfunction that causes chorea based on the excitement of the thalamus against the cerebral cortex.

In our patients, there was no chorea at the time of diagnosis of MMD; however, after bilateral bypass surgeries, there was new onset of chorea in adolescence. We believe that the cause was related to the changes in the pathways of the basal ganglia as the patients grew older. The nigrostriatal dopamine neurons and the components of the basal ganglia demonstrate marked age-related variations in their functions and morphologies [4]. Striatal indirect pathways are functionally immature in the childhood and attain
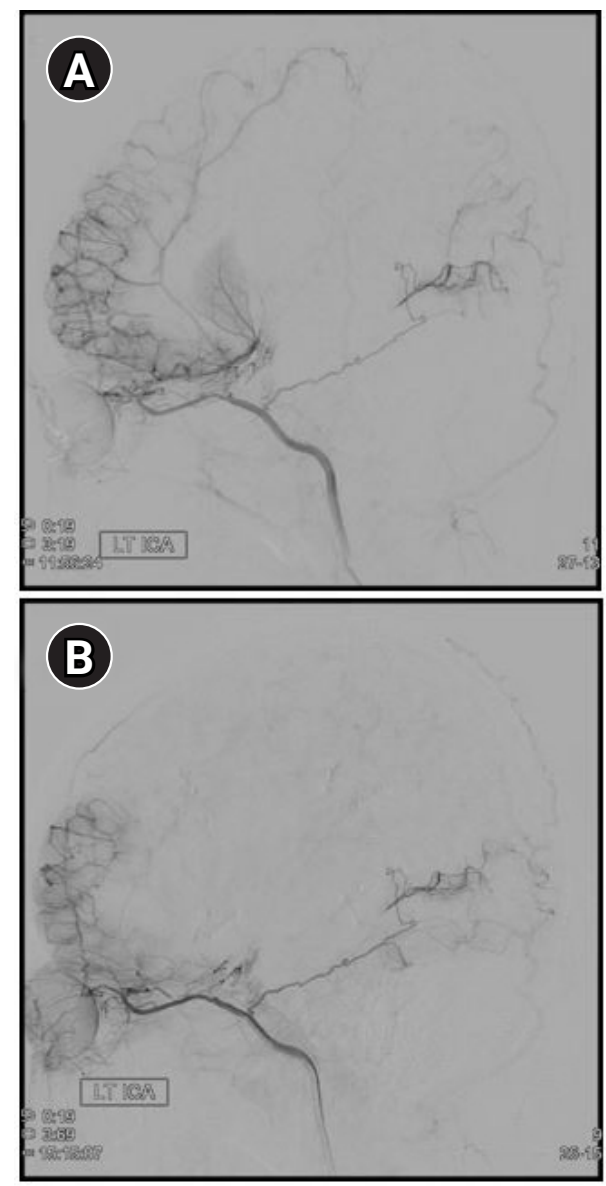

Fig. 2. Comparison of internal carotid angiography before and after surgical revascularization obtained in patient $B$. (A) Extensive network of collateral vessels that course through the basal ganglia before the bypass surgery. (B) Significant improvement in basal collateral after surgery.

adult levels approximately around the middle of the second decade. Therefore, even if there was a lesion in this area, if the structure of the lesion site was immature, the related symptoms would not appear initially. However, it is assumed that the associated symptoms appear when the lesion site matures with age. This is also consistent with the fact that the onset time of hemichorea in our patients was after the appearance of secondary sexual characteristics.

Cases of chorea are still difficult to manage because optimal therapy has not been established. Prior studies have reported that anticonvulsants including clonazepam, valproic acid, and carbamazepine can successfully control myoclonic hyperkinesia in huntington's disease and paroxysmal kinesigenic dyskinesia [5]. The mechanism of action of these agents in chorea is not well understood. Inducing neuronal excitability appears to be involved with an imbalance of both dopaminergic and GABAergic neurotransmissions. Clonazepam has antidopaminergic effects that are medi- 
ated predominantly by the central-type benzodiazepine receptors in the central nervous system via GABAergic mechanisms. The mechanism of action of valproic acid and carbamazepine is believed to be via increasing brain GABA levels either with sodium channel blockage or with GABA agonistic effects. So we decided to use anticonvulsants with antidopaminergic effect.

Patient A was started on clonazepam $0.5 \mathrm{mg}$ and valproic acid $600 \mathrm{mg}$ per day. A week after starting the medications, the patient demonstrated significant improvements in hemichorea; the frequency had decreased from approximately 20 to 10 episodes per day and the intensity was much weaker. Seven months later, his symptoms completely disappeared and the drug was stopped.

Patient B was started on clonazepam $0.5 \mathrm{mg}$ and carbamazepine $400 \mathrm{mg}$ per day. On the 4th day of starting the treatment, the patient demonstrated significant improvements; the right-hand tremor did not occur even once during the day or occurred weakly once or twice when there were many. After 4 months, the symptoms completely disappeared and the clonazepam was stopped. Since then, she is currently under outpatient follow-up.

In conclusion, our case report suggests that anticonvulsants with antidopaminergic effects can be considered as a treatment for new-onset chorea in patients who have undergone bilateral surgical revascularization in MMD. This study was approved by the Research Ethics Committee of Inha University Hospital (approval number: 2020-04-006). Written informed consent by the patients was waived due to a retrospective nature of our study.

\section{Conflicts of interest}

No potential conflict of interest relevant to this article was reported.

\section{ORCID}

Ga Hee Lee, https://orcid.org/0000-0002-9766-7914

Dong Hyun Kim, https://orcid.org/0000-0001-9883-0229

Young Se Kwon, https://orcid.org/0000-0003-4570-7037

\section{Author contribution}

Conceptualization: GHL and YSK. Data curation: GHL and YSK. Formal analysis: DHK and YSK. Funding acquisition: DHK and YSK. Methodology: GHL and YSK. Project administration: WRJ. Visualization: HSP. Writing-original draft: GHL. Writing-review \& editing: YSK.

\section{References}

1. Zheng W, Wanibuchi M, Onda T, Liu H, Koyanagi I, Fujimori $\mathrm{K}$, et al. A case of moyamoya disease presenting with chorea. Childs Nerv Syst 2006;22:274-8.

2. Berardelli A, Noth J, Thompson PD, Bollen EL, Curra A, Deuschl G, et al. Pathophysiology of chorea and bradykinesia in Huntington's disease. Mov Disord 1999;14:398-403.

3. Macpherson T, Morita M, Hikida T. Striatal direct and indirect pathways control decision-making behavior. Front Psychol 2014;5:1301.

4. Segawa M. Development of the nigrostriatal dopamine neuron and the pathways in the basal ganglia. Brain Dev 2000;22 Suppl $1:$ S1-4.

5. Chou IC, Lin SS, Lin WD, Wang CH, Chang YT, Tsai FJ, et al. Successful control with carbamazepine of family with paroxysmal kinesigenic dyskinesia of PRRT2 mutation. Biomedicine (Taipei) 2014;4:15. 